\title{
Efficient Energy and Position Aware Routing Protocol for Wireless Sensor Networks
}

\author{
Chaya Shivalingagowda ${ }^{1}$, Dr. P.V.Y Jayasree ${ }^{2}$, Dinesh.K. Sah ${ }^{3, *, 4}$ \\ ${ }^{1}$ Department of Electronics and Communication \\ Kalsekar Engineering College, New Panvel, 24105 Mumbai, India \\ [e-mail: chaya.ece@gmail.com] \\ ${ }^{2}$ Department of Electronics and Communication \\ GITAM university, Vizag, 531163 Andhrapradesh, India \\ [e-mail: ece.pjayasree@gmail.com] \\ ${ }^{3}$ Computer Science and Engineering Department \\ Indian institute of technology (Indian school of mines) Dhanbad, India \\ [e-mail: dksah.iitd@gmail.com] \\ ${ }^{4}$ Glean System, Gwalior India \\ [e-mail: ppal.nit@gmail.com] \\ *Corresponding author: Dinesh.K. Sah
}

Received August 14, 2019; revised November 12, 2019; revised December 20, 2019; accepted March 16, 2020; published May 31, 2020

\begin{abstract}
Reliable and secure data transmission in the application environment assisted by the wireless sensor network is one of the major challenges. Problem like blind forwarding and data inaccessibility affect the efficiency of overall infrastructure performance. This paper proposes routing protocol for forwarding and error recovery during packet loss. The same is achieved by energy and hops distance-based formulation of the routing mechanism. The reachability of the intermediate node to the source node is the major factor that helps in improving the lifetime of the network. On the other hand, intelligent hop selection increases the reliability over continuous data transmission. The number of hop count is factor of hop weight and available energy of the node. The comparison over the previous state of the art using QualNet-7.4 network simulator shows the effectiveness of proposed work in terms of overall energy conservation of network and reliable data delivery. The simulation results also show the elimination of blind forwarding and data inaccessibility.
\end{abstract}

Keywords: Sensor network, mobile sensor, path-planning, clustering, coverage hole, reliability 


\section{Introduction}

$\mathbf{W}_{\mathrm{i} \text { rest }}$ reless sensor networks (WSNs) is increasingly getting popular for the application environment due to its robust and low energy consumption attributes. A scenario like a battlefield, agriculture farm, underwater surveillance, etc., is some of the application environment that is benefited from WSNs monitoring mechanism [1, 2, 3]. Like other networking structure, such as mobile ad-hoc networks (MANET), vehicular network, body area network, etc., requires a set of networking protocol to establish the infrastructure. This paper focuses on the network layer aspect of WSNs. The routing protocols at the network layer of WSNs are classified as flat, hierarchical, location-based [4]. In flat-based routing protocol, all nodes co-ordinate at the same time for the transfer of data [5]. Some of the most widely discussed examples in literature are, sensor protocol for information via negotiation (SPIN) [6], Direct Diffusion (DD) [7], Rumor Routing (RR) [8] and Gradient-based Routing (GBR) [9]. These protocols, when used in a large network, cause energy consumption due to data generated by each node deployed in the target area. Hierarchical routing overcomes the drawback of flat routing by long-range data transmission by replacing the concept of the source to the destination from individual node to cluster-based node [10]. Cluster head collects the data from the node in its cluster and indirectly communicate the gathered data from an individual node to the destination node. Widely discussed approaches in this category are, low energy adaptive clustering hierarchy (LEACH) [11], Minimum energy communication network (MECH) [12] and power-efficient gathering in sensor information system (PEGASIS) [13]. In another approach, the routing decision is made based on the geographical location of the node, identified with the global positioning system (GPS) [14]. This approach helps in identifying the closest intermediate nodes in a route from source to destination, enabling the decision of duty cycling scheduling of other nodes [15]. Some of a good example is sequential assignment routing (SAR) [16], ad-hoc positioning system, and geographic adaptive fidelity. This proposal focuses on the drawback of Flat based routing and modifies the SPIN protocol approach to overcome the problem of Blind forwarding and Data in-accessibility. The key idea is energy and distance-based selection of intermediate node for data forwarding. In each step, the protocol identifies the closest node with the maximum remaining energy for forwarding the data. The proposed approach also balances the energy consumption due to data forwarding, eliminating the problem of data inaccessibility due to the coverage hole. The results are generated and compared in QualNet7.4 network simulator and are compared with SPIN and Direct Diffusion routing protocol. The key research contribution of the proposal is the identification of the shortest route from source to sink and is determined by identifying the remaining energy and hop count of source node from the sink node. The proposal eliminates the problem of blind forwarding and data inaccessibility and also balances the energy consumption of an individual node in the network avoiding the problem of hot-spot. The rest of the paper is organized as follows. Section 2 presents the system model and problem definition. In section 3, related work has given for understanding of the problem. In section 4, problem formulation has been discussed followed by section 5 , in which proposed work has given. In the performance section, the approaches with its pros and cons are discussed. Moreover, in the performance analysis section, the assertion of the performances of individual's parameters of the proposal over the existing approach has discussed with each performance metrics. The given protocols being evaluated through simulation extensive simulation and have been discussed in section 6. Finally, conclusions are drawn in section 7. 


\section{System model}

\subsection{Scenario Description}

The underlying target environment $A$ is of dimension $\left(A_{x} \times A_{y}\right)$. A mixture $P$ of stationary nodes, are deployed in target area $A$; randomly deployed sensor node location is identified as $p_{i}=\left(p_{x}, p_{y}\right)$. Where coordinates of latitude and longitude position are given by $p_{x}$ and $p_{y}$, respectively. QualNet 7.4 network simulator is used for creating simulation scenarios [17], for tractability, $A$ is subdivide in grid of square cells of size . Thus, $A$ is modeled as a lattice $H$ of size $H_{x} \times H_{y}$, where $H_{x}=\left\lceil\frac{A_{x}}{d}\right\rceil$ and $H_{y}=\left\lceil\frac{A_{y}}{d}\right\rceil$. After modeling the target area into grid of square cell, sensor node $p_{i}$ situated at $\left(p_{x}, p_{y}\right)$ is in the cell $c_{i}=\left(\left\lceil\frac{x_{i}}{d}\right\rceil,\left\lceil\frac{y_{i}}{d}\right\rceil\right) \in H$. Sensing model of node in terms of isotropic and communication aspect is presented as circle of radius $r_{s}$ and $r_{c}$, respectively, where $r_{s}<r_{c}$.

Table 1. Abbreviation used in text

\begin{tabular}{|c|l|c|l|}
\hline Abbreviation & Description & Abbreviation & Description \\
\hline$P_{i}$ & Sensor Node & $E_{t x}(l, d)$ & Energy consumes by the transmitter \\
\hline$r_{s}$ & Sensing Range & $E_{r x}(l)$ & Energy consumes by the receiver \\
\hline$r_{c}$ & $\begin{array}{l}\text { Communication } \\
\text { Range }\end{array}$ & $E_{\text {coms }}$ & $\begin{array}{l}\text { Energy consumes by the transceiver } \\
\text { circuit }\end{array}$ \\
\hline$T$ & Time & $€$ & Channel fading constant \\
\hline$H$ & Lattice & Hop_id & Hop identification \\
\hline$A$ & Target Area & INS_packet & Initialization packet \\
\hline$C$ & Cell & $\begin{array}{l}\text { Square cell } \\
\text { dimension }\end{array}$ & Packet delivery ration \\
\hline$d$ & \multicolumn{2}{|l}{} & End-to-end packet delivery \\
\hline
\end{tabular}

Definition 2.1 Two sensor nodes are in neighborhood of each other, if they lies in the communication range of each other, i.e., $\left\|p_{i}-p_{j}\right\| \leq r_{c}$ (Fig. 1).

A set of node can only communicate to a node if they are in communication rage of the $r_{c}$ target node. Therefore, the possible number of neighboring nodes to a node at a given time $k T$, is given as $H_{r_{c}}(k)=\left\{j:\left\|p_{i}(k)-p_{j}(k)\right\| \leqslant r_{c}, j \leqslant P\right\}$.

Definition 2.2 A generic node $p_{i}$ with sensing range $r_{s}$ at time $k T$ covers a subset $H_{i}(k) \in A$ of cells, and is given in Equn.1:

$$
H_{i}(k)=\left\{(w, q):\left(p-\left\lceil\frac{x_{i}(k)}{d}\right\rceil^{2}\right)+\left(p-\left\lceil\frac{y_{i}(k)}{d}\right\rceil^{2}\right) \leqslant\left\lceil\frac{r_{s}}{d}\right\rceil\right\}
$$

Where $w$ and $q$ are numbers with $1 \leqslant w \leqslant H_{x}$ and $1 \leqslant q \leqslant H_{y}$. Note that $H_{i}(k)$ relies upon $\mathrm{k}$ for $M N$ only.

\subsection{Energy Model}

The energy consumption by a node is divided in two categories one due to communication due to transceiver circuitry and another due to transducer unit of sensor of the node. Since the path overlapping increases the communication overhead in network, only energy consumption due to transceiver circuitry is considered. 
Definition 2.3 Energy consumption by transceiver circuitry in a node is given in Equn.2:

$$
E_{\text {coms }}(l, d)=E_{t x}(l, d)+E_{r x}(l)
$$

Where $E_{t x}(l, d)$ and $E_{r x}(l)$ is the energy consumed by transmitter and receiver unit, respectively, while transmitting 1 bit message and is given by Equn. 3 and 4 .

$$
\begin{aligned}
& E_{t x}(l, d)=E_{C c t}(l)+E_{a m p}(l, d) \\
& E_{r x}(l)=E_{C c t}(l)=l \times E_{C c t}
\end{aligned}
$$

The distance $d$ between receiver node $p_{j}$ and transmitter node $p_{i}$ is used to identify $E_{t x}(l, d)$ model at a given instance $t$, and is given by Equn.5.

$$
E_{t x}(l, d)= \begin{cases}l \times E_{C c t}+l \times \varepsilon_{f s} \times d^{2} & d<d_{0} \\ l \times E_{C c t}+l \times \varepsilon_{m p} \times d^{4} & d \geq d_{0}\end{cases}
$$

Where $\varepsilon_{f s}$ and $\varepsilon_{m p}$ represent free space and multi-path fading channel model, respectively. If $d$ is greater than given threshold $d_{0}=\sqrt{\frac{\varepsilon_{f s}}{\varepsilon_{m p}}}$ then multi-path fading channel model is utilize, otherwise, free-space model is used.

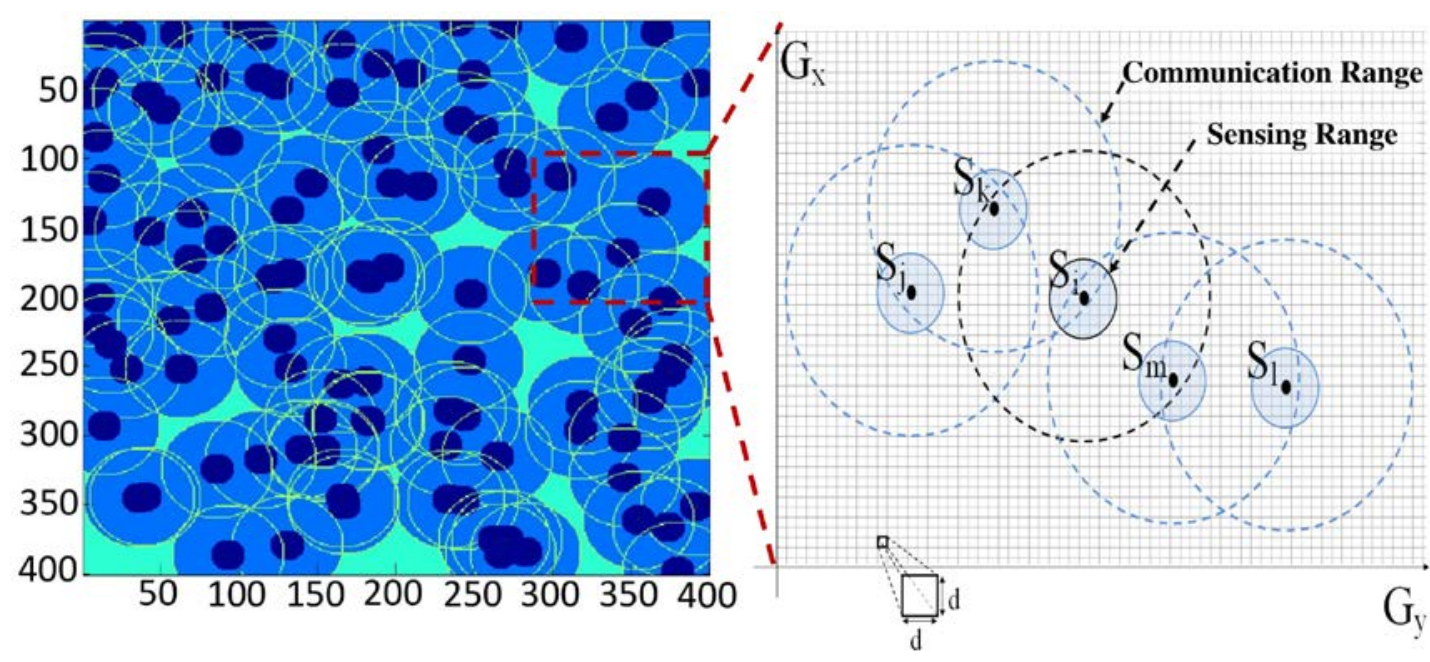

Fig. 1. Sensor nodes in Simulation scenario along with their sensing range $\boldsymbol{r}_{\boldsymbol{s}}$ (shown in black line arround) and communication range $\boldsymbol{r}_{\boldsymbol{c}}$ (shown in dotted line arround) representation (color should be used for this Fig. 1 in print).

\subsection{Communication Stack}

RIME communication stack in contiki operating system is used to establish multi-hop communication between nodes and the sink [18]. RIME is a custom lightweight networking protocol designed for the low-power wireless network and is a part of the Contiki operating system. All real-time and simulation scenario design in QUALNET-7.4 are using RIME communication stack at data link layer. 


\section{Related Work}

Flooding is the most effortless approach to advance Intrigue packets on the wireless medium. Such a methodology has the prudence of effortlessness and well faces circumstances in which start to finish way set up and support are troublesome and expensive, for example, in powerful ad hoc conditions and with asset obliged devices. Flooding encourages content sharing in the network; actually, a node overhearing a few data of intrigue mentioned by different nodes can get to it without an express solicitation. This diminishes the quantity of transmissions and recoveries the node's energy. However, flooding on a broadcast medium must be manoeuvred carefully and controlled to maintain a strategic distance from the broadcast storm [1]. To neutralize packets repetition and collisions, solutions in the writing, for the most part, depend on distributed packet concealment systems. The essential thought is that a node defers the packet forwarding while at the same time overhearing the channel and, eventually, drops the packet in the event that it hears the packet transmitted by a neighbour $[2,3]$. Distance-based, slotted random, or simply random concede procedures can be actualized. In [4], a set of timers is utilized to help Data broadcasting in vehicular situations. In particular, a crash shirking timer is utilized by neighbouring autos that at the same time get an Enthusiasm for road turned parking lot information, to reschedule Data broadcasting on various occasions. A comparable methodology is pursued in $[5,6]$, where distinctive concede timers are utilized for Intrigue and Data forwarding in order to limit the crash likelihood and organize Data over Interests. In any case, a visually impaired controlled flooding based on the previously mentioned basic countermeasures doesn't generally ensure that (i) the best nodes are chosen to advance packets, and that (ii) overhearing stays away from packet collisions.

The most widely explored category of routing strategy is flat-based routing is Flat based routing, each node co-ordinate based on the same set of instruction to perform the communication and sensing task, i.e., the role of each node is similar from communication and sense point of view and hence termed as Data-centric approach [19]. In data-centric methods sink sends an inquiry to an individual node in a given region and in response node reply via multi-hop communication. The response can be the sensed data or network information. SPIN is the most commonly referred data-centric approach [20]. SPIN protocol over flooding based strategy overcomes the problem of data burst via a negotiation-based approach [21]. A node before transmitting data negotiates with the neighbor nodes and then forwards data to an interested node only. The following section discusses SPIN, Direct diffusion, and rumor routing protocol. Sensor protocol information via negotiation (SPIN): the key concept of SPIN is metadata exchange between the nodes in one-hop communication before the transfer of actual data. Meta-data is shared with the help of a small advertisement packet, which consumes less of network resources. Each node maintains the list of meta-data of data generated by sensing or data forwarded previously during multi-hop communication. After receiving the ADV packet holding a meta-data neighbor node first compare the list of meta-data with the information present in the ADV packet. If similar data is their neighbor node does not reply, else, it will reply with a request packet REQ. SPIN Metadata strategy overcomes the problem of data flooding in-network by data redundancy with the help of negotiation operation, which saves much of network energy and time. SPIN works on three message exchange principal, ADV, REQ, and DATA. First, the node transmits broadcast ADV packet encapsulating meta-data to the one-hop neighbor node. In response, an interested neighbor node sends the REQ packet and then the node sends the original data. SPIN is not suitable for the application environment which requires data security or 
reliability, i.e., tactical networks or health monitoring.

Directed Diffusion (DD) is another protocol that is created after the SPIN. Directed Diffusion goes for diffusing data through sensor nodes by using a naming plan for the data [22]. DD uses attribute-value sets for the data and furthermore inquiries the sensors on the interesting premise by utilizing those sets. To make a query, an intrigue is characterized by utilizing a rundown of attribute-value pair, for example, objects name, topographical territory, term, interim, and so forth. This intrigue is further broadcast by a sink through its neighbors. Each hub that gets this intrigue can do storing for later use. The nodes additionally had the adaptability to do in-network data collection. The interests in the stores are then used to contrast the data got and the values in the interests. The intrigue passage additionally contains different angle fields. This inclination might be an answer connect to a neighbor from which the intrigue was gotten. Henceforth, by using interest and slopes, paths are set up among sink and sources. A few paths can be set up with the goal that one of them is chosen by support. DD is much energy proficient than others since it is on interest and there is no requirement for keeping up worldwide network topology. Be that as it may, it can't be connected to all sensor network applications since it depends on a query-driven data conveyance show.

Rumor Routing (RR) is a trade-off between flooding inquiries and flooding event warnings [23]. The primary arrangement of this protocol is to make paths that prompt each event, dissimilar to event flooding which makes a network-wide gradient field. Along these lines, on the off chance that an inquiry is produced it very well may be then sent on a random walk till it finds the desired path, instead of flooding it all through the network. When the required path is found it tends to be additionally steered specifically to the event. Then again, if the path can't be discovered, the application may attempt re-presenting the inquiry or flooding it. The RR might be a decent technique for conveying questions to the event in huge networks.

\section{Problem formulation}

The SPIN protocol is suited for medium size WSNs like, agricultural monitoring, battel field monitoring.

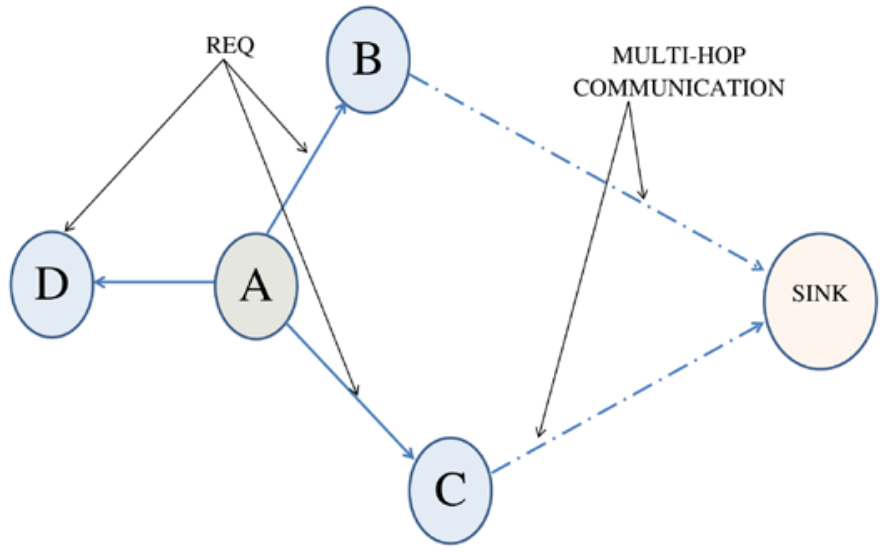

Fig. 2.a. Blind forwarding overview 


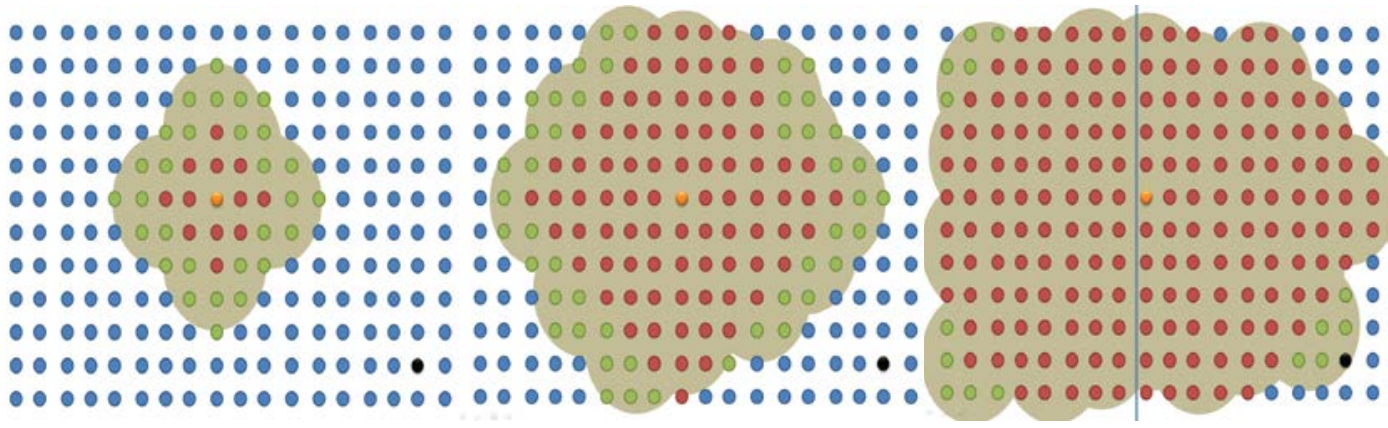

Fig. 2.b. Data dissemination instances in SPIN-BC at different instance of time in a network of 204 sensor nodes.

The SPIN protocol is a versatile communication and data-centric routing protocol. To keep away from the rise of the explosion of data and some overlap in flooding, the node interacts with one another through utilizing meta-data before transmitting the actual information. Therefore, it prevails with regards to staying away from the blind utilization of resources and avoid "implosion" and "overlap" issue in the flooding protocol. However, it can not overcome the problem of Blind forwarding and data inaccessibility. Blinforwarding is the problem that occurs due to the transmission or flow of data in the direction away from the sink node. On the other hand, data inaccessibility occurs when a node has data to send but all it's neighbor node is dead due to total energy consumption. Full insight of blind forwarding and data inaccessibility is given in the following section.

\subsection{Blind forwarding and Data inaccessibility}

All those nodes who do not have a meta-data record for a packet received via ADV message, in their list participate in data forwarding via the REQ packet irrespective of their reachability to sink node [24]. Nodes having data to transmit broadcast ADV messages to all neighbor nodes. For example in Fig. 2.a node A will broadcast ADV message carrying metadata information of data destined to sink node to all its one-hop neighbor. Nodes B, C, and D will receive the ADV packet and respond via REQ message: given none of them have similar meta-data information in their list. Hear data forwarding to node D is of no use because either the data will never reach the sink or take a long route in terms of hop to reache the sink and this phenomenon is termed as blind forwarding. A broder view of problem in a network of 240 sensor node is shown in Fig. 2.a and 2.b. In Fig. 2.b red represent the node forwarded the actual data, green represent the node received the ADV message and black and orange represent the sink and the source node. From the Fig. 3 , it can be concluded that data dissemination to nodes left of the source node is of no use since those packets will never reach the destination and are miss utilization of network resource. In another real-time scenario of home-automation has shown in Fig. 3, where the node is deployed on the ground floor, first floor, second floor, third floor and roof. In the existing approach, the data is forwarded to all the neighbour nodes. For example, if a node at the third floor wanted to send data to the sink node at the ground floor, it will flood the data in all direction including the node on the fourth floor. However, the data forwarded to the fourth floor and further to the sensor at the roof will never make it to the sink at the ground floor. In the proposed approach the data is disseminated only to the node closer to sink node and hence in Fig. 3 the source node at the third floor will only forward data to the sensor at the third floor or to the second 
floor depending on the communication range of the source node. Data inaccessibility is the event when the source node is unable to forward data to destination node [25]. The dominating cause is the depletion of node energy and is the reason for the rejection of the ADV packet. In a scenario where all the nodes in the path to destination reject the ADV message causes the inaccessibility of data. For example, if all the data communication to the sink node follows the same source to destination path, then the energy depletion rate of the node in the path also increases leading to multiple node termination along the path. The later event is due to the uneven load balancing of a transmitted packet in the network and is also termed as the hotspot. However, there can be other reasons specific to an underlying scenario which causes the termination of the node, for example, in forest surveillance application scenario node can be deactivated due to malfunction because of heavy rainfall or high-speed wind.
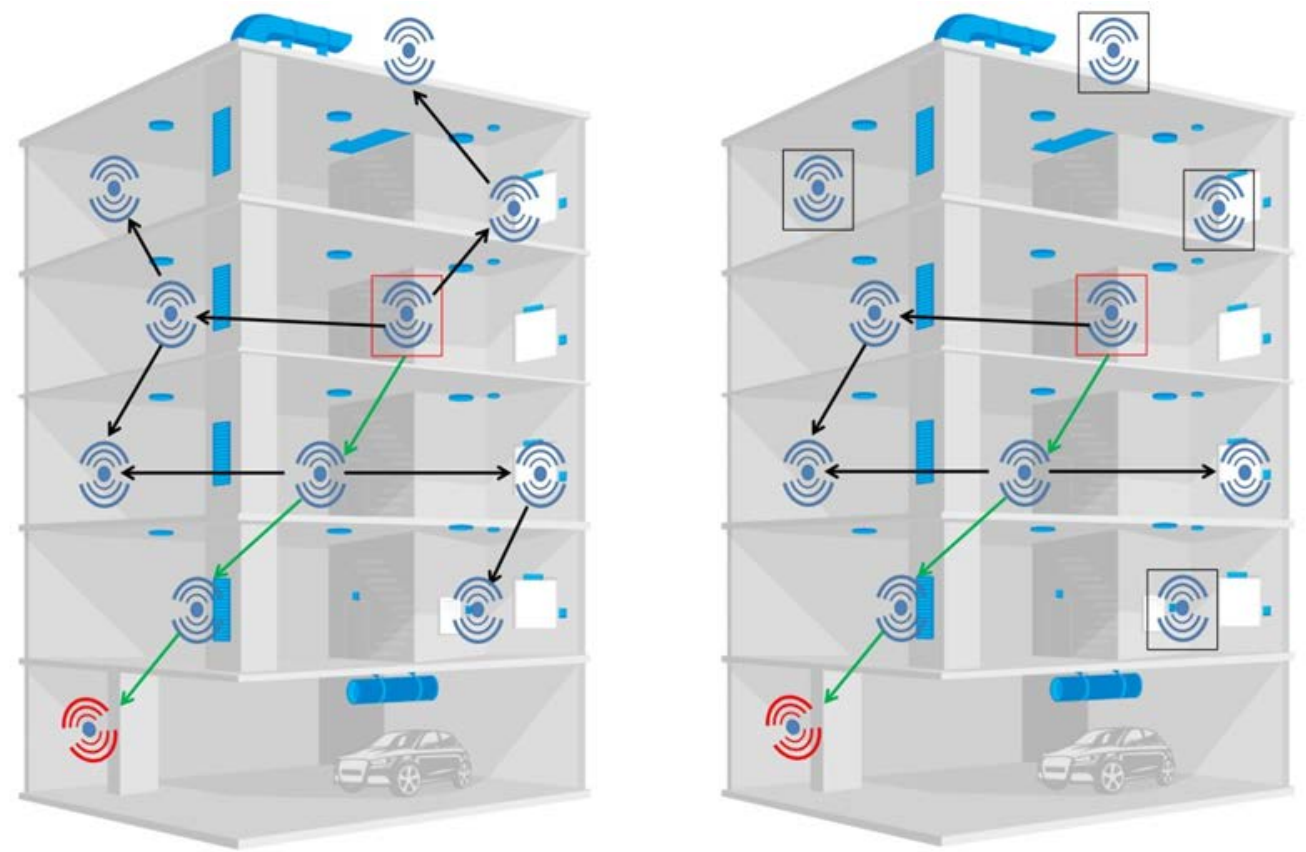

Fig. 3. Problem formulation for home automation scenario: (a) existing approaches and (b) Proposed model

\section{Proposed Work}

\subsection{Initial assumption}

We have made some assumptions such as the initial energy of all the nodes at initialization is the same. All network node generates data packet randomly and is destined to sink node. Since the multi-hop network topology is followed a node can accept packet only when the buffer of the receiving packet is empty. A buffer size of $1.25 \mathrm{MB}$ capable of holding 10 packets at a time are initialized at the time time of network setup Two nodes can communicate via full-duplex mode. Full duplex mode, communication between two nodes in a mesh topology is achieved by full-duplex communication; given the energy, decimation is not affected by the communication protocol. The radio propagation model of the node is modeled based on the CC2538 low power system on chip micro-controller. Signal interference between node $n_{i}$ and $n_{j}$ because of other nodes is given as: In an undirected graph $\mathrm{G}(\mathrm{V}, \mathrm{E})$, with $\mathrm{V}$ vertices and $\mathrm{E}$ edges and given cost function $w: V \times V->R^{+}$, the 
receiver/sender interference at vertex $v_{p}$ is the $\left|v_{i}\right|$, where $I=1,2,3, \ldots . n|n<| v \mid$, in the transmission range of $v_{i}$. In the initial stage of the deployment, all nodes are reachable to the sink node. Nodes consume the same amount of energy for sensing and communication operation and should be above the threshold defined during network setup.

\subsection{Working Mechanism}

The proposal follows four stages to construct a source-to-destination route, and are as follows: (1) Node to sink distance calculation (HOP_ID), (2) Meta-data advertisement phase, (3) Data request phase and (4) Actual data transmission phase as shown in Fig. 4. The nose to sink distance in terms of a number of a hop count to reach the sink $H O P_{-} I D$ is calculated for all nodes in the network. It is assumed that nodes in the network are static and don't change their position throughout their lifetime . In advertisement phase source node $n_{i}$ broadcasts the meta-data of original data to be transmitted. All the neighbor $n_{j}$ of $n_{i}$ after receiving the ADV packet evaluate the energy level based on the fact that they are capable of receiving and then forwarding the received data. If the current energy value of an individual neighbor node $E N G_{c u r}^{n_{j}}$ is above the predefined threshold and its $H O P_{-} I D\left(n_{j}\right)$ is less than $H O P_{-} I D\left(n_{i}\right)$ it will move to request phase of the proposal. In request phase node neighbor node $n_{j}$ transmit a request message REQ along with $E N G_{c u r}^{n_{j}}$ and $\operatorname{HOP} I D\left(n_{j}\right)$ to source node. In data transmission phase source node $n_{i}$ sorts the REQ message based on their $E N G_{c u r}^{n_{j}}$ and the node with high energy value is selected for data transmission. Node-to-sink distance calculation: the process is executed after the initial deployment of the WSNs and since the node is static the parameter remains the same throughout the lifetime. In the case of WSN deployed for an environment where there is a possibility of node movement due to an intruder or environmental condition node can change their positions. In a given circumstance, the Node-to-sink distance calculation can be scheduled after a given interval of time or at the occurrence of an event. The sink broadcast the initialization packet (INS_PACKET) with attribute Node_ID and $H O P_{-} I D$, and is received by all hone hop neighbor. The Node_ID is the MAC address of the individual node and $H_{O} P_{-} I D$ is a counter initialized to a larger value. In proposed work initially the $H_{O P} I D$ is set to 10 since the maximum hop count observed after building the spanning tree of given deployment is 8 .

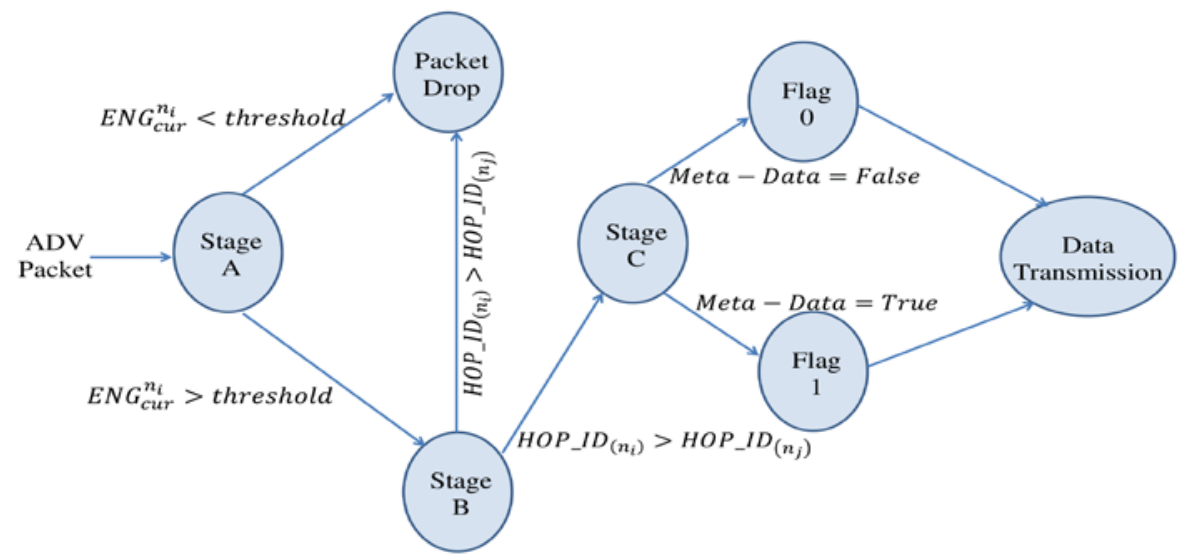

Fig. 4. Flow chart for proposed mechanism. 
The node $n_{j}$ after receiving the (INS_PACKET) from $n_{i}$ it compare the $H O P_{-} I D\left(n_{i}\right)$ to its $H_{O P} I D\left(n_{j}\right)$ and if $H_{O} P_{-} I D\left(n_{i}\right)<=H_{O} P_{-} I D\left(n_{j}\right)$, it save the Node $I D\left(n_{i}\right)$ and increment the counter HOP_ID $\left(n_{j}\right)$ and forward the (INS_PACKET) to its one hop neighbor nodes. The re-broadcasted (INS_PACKET) replaces the HOP_ID value of its own $H_{O_{-}} P_{-} I D\left(n_{j}\right)$ value. The whole process is mathematically modeled by Equn.6 and 7.

$$
\begin{aligned}
& H O P_{-} I D_{n_{j}}(t+1)= \begin{cases}H O P_{-} I D_{n_{j}}^{\text {min }} & \text { if INS_packet }==\text { true } \\
H O P_{-} I D_{n_{j}}(t) & \text { otherwise }\end{cases} \\
& H O P_{-} I D_{n_{j}}^{\min }=\min \left\{H O P_{-} I D_{n_{i}}, H O P_{-} I D_{n_{j}} \mid j \in 1, m\right\}
\end{aligned}
$$

Here, $\min \left\{H O P_{-} I D_{n_{j}}, H O P_{-} I D_{n_{i}}\right\}$ is the smallest count for hop distance from sink and $m$ is the total number of initialization packet received by an individual node. The process of hop distance calculation continues until each node in WSNs receives the initialization packet at least once.

Advertising Phase: when a node $n_{i}$ has data to transmit, collected either by itself or received by the neighbor node, it broadcast an ADV message to all its one-hop neighbor node, it broadcast an ADV message to all its one-hop neighbor. The main goal is to select the fittest neighbor $n_{j}$ among the given set of neighbors. The ADV message consists of metadata of data to be transmitted.

Request Phase: A node $n_{j}$ after receiving an ADV packet evaluates its energy based on the total energy required to process all three phases of the data communication process. The required energy level is presented as a predefined threshold value thresh $\left(n_{i}\right)$, and if the $E N G_{\text {cur }}^{n_{j}}$ is above this threshold it compares the HOP_ID $\left(n_{i}\right)$ over source node to its own $H O P_{-} I D\left(n_{j}\right)$. If the HOP_ID $\left(n_{j}\right)>H_{O} P_{-} I D\left(n_{i}\right)$ it moves forward to compare the metadata in ADV packet to list of meta-data in its record. If the match is found in the algorithm.1, a FLAG is set to 1 , else FLAG is set to 0 .

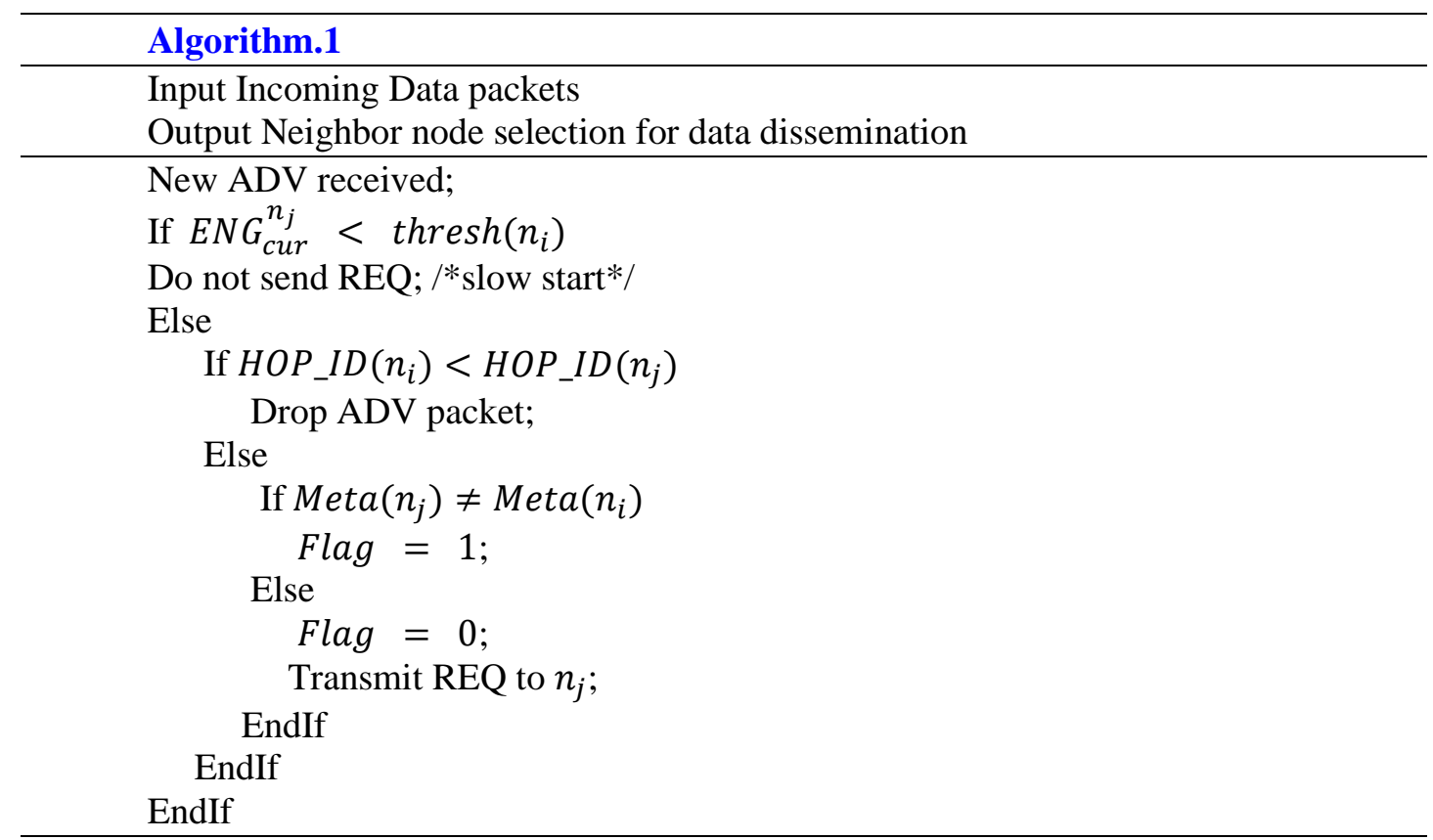


In next step node send a $R E Q\left(n_{j}\right)$ packet encapsulation node current energy $E N G_{c u r}^{n_{j}}$, hop distance from sink HOP_ID $\left(n_{j}\right)$ and FLAG value. The whole process is mathematically modeled by Equn.8, 9 and 10 .

$$
\begin{aligned}
& A D V_{\text {reply }}^{n_{j}}(\text { energy })=\left\{\begin{array}{ll}
1 & \text { if Energy } y_{\text {current }}^{n_{j}} \\
0 & \text { otherwise }
\end{array}\right. \text { Threshold } \\
& A D V_{\text {reply }}^{n_{j}}\left(\text { Hop }_{\text {distance }}\right)=\left\{\begin{array}{ll}
1 & \text { if Hop } p_{\text {distance }}^{n_{j}} \\
0 & \text { otherwise }
\end{array}<\right.\text { Hop distance } \\
& A D V_{\text {reply }}^{n_{j}}(F L A G)= \begin{cases}1 & \text { if Hop } n_{n_{j}}(\text { meta }- \text { data })=n_{i}(\text { meta }- \text { data }) \\
0 & \text { otherwise }\end{cases}
\end{aligned}
$$

Data Transmission Phase: In this phase the source node $n_{i}$ separate the $R E Q\left(n_{j}\right) \mid j \in m$, on the basis of their FLAG values. Separate list $L I S T_{0}$ and $L I S T_{1}$ are formed for REQ packet having FLAG value 0 and 1, respectively. The main goal of this strategy is to bypass those neighbor nodes which already have the data received by the current node and minimizes the miss utilization of WSNs resources. The $n_{i}$ short the element of $L I S T_{0}$ and $L I S T_{1}$ in decreasing order of their energy value. The source node select the top node $n_{j}$ or in other word the node with maximum energy from $L I S T_{0}$. If in case $L I S T_{0}$ is empty the top node from $L I S T_{1}$ is selected. The selected node is then paired to send the actual data. The whole process can be summarized as follow:

1. Node $n_{i}$ have data to transmit.

2. $n_{i}$ transmit $\mathrm{ADV}$ packet encapsulating meta-data to one hop neighbor nodes.

3. $n_{j}$ receives the ADV packet.

4. If $E N G_{c u r}^{n_{j}}>$ threshold go to next step, else drop the ADV packet.

5. If $H O P_{-} I D\left(n_{i}\right)<H O P_{-} I D\left(n_{j}\right)$ go to next step, else drop the ADV packet.

6. If meta $-\operatorname{data}(A D V)=$ meta $-\operatorname{data}\left(n_{j}\right)$, set $F L A G=1$ otherwise, $F L A G=0$

7. Transmit REQ(FLAG, HOP_ID $\left.\left(n_{j}\right), E N G_{c u r}^{n_{j}}\right)$

8. $n_{i}$ receives the $R E Q\left(F L A G, H O P_{-} I D\left(n_{j}\right), E N G_{c u r}^{n_{j}}\right)$

9. Create $L I S T_{0}$ and $L I S T_{1}$ for $R E Q\left(F L A G_{0}\right)$ and $R E Q\left(F L A G_{1}\right)$

10. Short $L I S T_{0}$ and $L I S T_{1}$, based on their $E N G_{c u r}^{n_{j}}$ in descending order.

11. If $L I S T_{0}==N u l l$, select the top element, otherwise, select $L I S T_{1}$ top element.

12. Transmit actual data to selected element.

\section{Complexity Analysis:}

The time complexity of proposed work is equivalent to other approaches considered in the result section of the proposal. However, the complexity in terms of energy utilization in computation is reduced to one fourth. The reasoning for the reduction of computation to fourth of the existing is as since, the packets are directed in only one quadrant of the target area. In the worst-case scenario, the sender node at the top left most, considering that the sink is located in the right bottom corner of the target area. In this scenario, the metadata will be flooded to all the node. In a best-case scenario, the node is in the middle or in the quadrant in which the sink is located. 

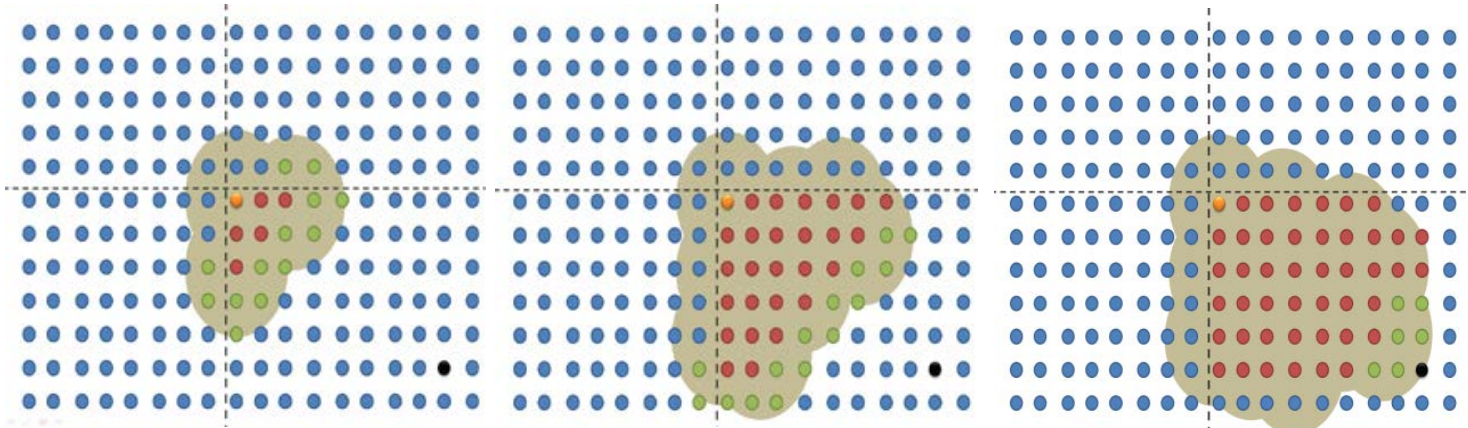

Fig. 5. Data dissemination instances proposed protocol at different instance of time in a network of 204 sensor nodes.

\section{Performance analysis}

Performance evaluated by scenario modeling in the QualNet-7.4 network simulator . The performance is evaluated over: (1) Total energy consumption of network, (2) Number of alive nodes after a given number of iteration and (3) Throughput of the network. The system model for all the simulation scenarios are presented in section 2 . In all the presented scenario the network is composed of $300 \times 300 \mathrm{~m}^{2}$ and $150 \times 150 \mathrm{~m}^{2}$. Two different scenarios are considered to vary the density or count for number of sensor node with in the communication range of a node. High neighbor node density provides higher degree of path selection approach which causes more data dissemination and energy consumption in network. The simulation results are performed over total energy consumption, number of alive node, average end to end delay, average and packet delivery ratio. The same is performed in WSN of 150 and 300 node infrastructures and is permutated over region of $300 X 300 \mathrm{~m}^{2}$ and $500 X 500 \mathrm{~m}^{2}$. Further, throughput evaluation is performed over low, medium and high traffic rate in WSNs of 500 sensor nodes.

\subsection{Result discussion}

In Fig. 5, data dissemination instances proposed protocol at different instance of time in a network of 204 sensor nodes. Moreover, the comparision in Fig. 6 and 7 shows the energy consumption by SPIN-BC, SPIN-RL, M-SPIN and Proposed approach. It can be concluded that the overall energy consumed by proposed protocol is low. The difference in energy consumption in Fig. 6 is comparatively more than Fig. 7, it is because the density of node distribution in per unit area is resulting in more neighbor node per node. Increased rate of neighbor node adds more path option for data dissemination resulting in excess energy consumption. The number of alive node is good measure of network lifetime. Fig. 8 and 9 presents the number of alive node after $\mathbf{1 0}^{\mathbf{3}}$ request completion at sink node in target area of dimension $\mathbf{1 5 0} \times \mathbf{1 5 0} \mathrm{m}^{2}$ and $\mathbf{3 0 0} \times \mathbf{3 0 0} \mathrm{m}^{2}$. Energy consumption by an individual node is presented by equation and is modeled in QualNet-7.4 network simulator. In Fig. 8, the frequency of dying node is higher in comparison to Fig. 9 since the rate of data disseminated in network of target are $\left(\mathbf{1 5 0} \times \mathbf{1 5 0} \mathbf{~ m}^{\mathbf{2}}\right)$ is much more than in target area $(\mathbf{3 0 0} \times$ $\mathbf{3 0 0} \mathrm{m}^{2}$ ) where the nodes are distributed sparsely in the region. The PDR evaluation is based on formula given by Equn.11, respectively. 


$$
P D R=\frac{\text { Packet_Delivered }}{\text { Packet_Sent }}
$$

It is assumed that all links between source to destination are homogeneous, and based on this fact EEPD is calculated as in Equn.12:

$$
E E P D=K\left(D_{\text {proc }}+D_{\text {queue }}+D_{\text {trans }}+D_{\text {prop }}\right)
$$

Where $K$ is the number of intermediate nodes, $D_{\text {proc }}$ is processing delay at a node, $D_{\text {queue }}$ is queuing delay and $D_{\text {prop }}$ is propagation delay. The average end-to-end packet delay (EEPD) is analyzed in all four cases and the results are shown by Fig. 10. the comparison for Packet delivery Ratio is presented by Fig. 11. The analysis is performed over the target area of dimension $300 \times 300 \mathrm{~m}^{2}$. In Fig. 9 and Fig. 10, we can see that our proposal outperform the existing approaches. One of the major advantages of having the protocol specific to uniform topology is that the data forwarding nodes over multihop communication can be more accumulated. The selection strategy of forwarding node is more deterministic in our approach over other existing protocols. As we can observe from Fig. 2.a and Fig. 5, our proposal has opted more nodes towards sink for data forwarding over the existing approaches. The same advantage can be observed in Fig. 11 also as energy consumption is less, over the time number of node alive is also greater than the existing approaches.

Network throughput in two scenarios is analyzed to measure data reliability. Throughput is the measure over source to sink connection and is given by Equn.13 and 14 .

$$
\text { Throughput }=\frac{S_{\text {Received }}}{N_{\text {transmit }}}
$$

Where $N_{\text {transmit }}$ is the total number of packet transmitted by nodes in network and $S_{\text {receive }}$ is the number of packet actually received by sink node. Throughput over different source to destination connection is presented in Fig. 12 and it can be observed that the proposed protocol overcomes other approaches. Throughput is calculated over the rate of successful packet transmission over the medium and is measured as the number of packets received in a time slot.

$$
\text { Throughput }=\frac{\text { sucessfull_packet_transmission }}{\text { total_packet_transmission }}
$$

The simulation consists of 100, 250 and 500 sensor nodes uniformaly deployed in a circular target area of radius 150 meters. Base station is fixed at the center of circular region. The results are generated for a simulation run of 2 hours and are compared for three different traffic rate as low, medium and high. The traffic rate is varied based on number of packet transmission by individual node. Fig. 13,14 and 15 shows the result for average throughput for low, medium and high traffic rate respectively. In our approach, the meta-data of nodes helps to select the nodes for data forwarding which have high energy storage. In Fig. 13, 14 and $\mathbf{1 5}$ the average throughput in three different scenarios is compared over the proposed algorithm, M-SPIN, SPIN-BC and SPIN-RL. The results are compared over low, medium and high traffic scenarios. From Fig. 13-15, it can be concluded that the packet loss ratio in all the three scenarios in the proposed approach is less than other proposals. In Fig. 15 the packet loss ratio is high compared to low and medium traffic scenario but the proposed approach tend to show better results than M-SPIN, SPIN-BC and SPIN-RL. It does not only 
enhance the throughput but also reduces the packet drop from individuals nodes. Once, on an average the forwarding nodes alive and that too in the direction of the sink, it enhances the throughput as well as PDR which can be observed in Fig. 15, 17 and 18. The packet drop ratio is the measure of the reliability of the network and is affected by the routing decisions made by the underlying network protocol. In Equn.15, the packet drop ratio value has calculated to analyze the network performance in term of packet drop.

$$
\text { packet_drop_ratio }=\frac{\text { total_number_of_packet_drop }}{\text { total_packet_transmission }}
$$

The following simulations are conducted to show the effectiveness of proposed protocol over protocol in high traffic scenarios. The effect is emphasized by performing subsequent simulation over low and medium traffic rates. The simulation consists of 100, 250 and 500 sensor nodes uniformaly deployed in a circular target area of radius $=150$ meters. The base station is fixed at the center of the circular region. The results are generated for a simulation run of 2 hours and are compared for three different traffic rates low, medium and high. Fig. 16, 17 and 18 shows the result for packet loss ratio for low, medium and high traffic rate respectively.

\subsection{Statistical validation: Analysis of variance (ANOVA)}

We have evaluated the proposed protocol based on two important metrics (throughput and packet delivery ratio) which are critical to WSNs. To validate the statistical significance of the proposed method we are using one of the popular test suggested in [29] named as analysis of variance (ANOVA) test. We are performing ANOVA test on two parameters which is throughput and PDR value and the resulting graph shown in Fig. 20 and 21. Further, the result of the hypothesis test has compared with the existing proposal to prove the statistical significance of the simulation result. The ANOVA test, we consider the mean of two given sample or scheme and compare whether it is the same or not. In general, an ANOVA test can provide the insight that whether the null hypothesis $\left(H_{\text {null }}\right)$ which states that mean of two or more method for the chosen set of samples are same and therefore should be rejected as mentioned in Equn.16. The parameters used in ANOVA test provide the outcomes in the form of F-statistic. The $H_{\text {null }}$ will be rejected only when the given two conditions are satisfied.

(i) The $p$-value should be less than the significance level.

(ii) The value of $F$-statistic must be higher than the $F$-critical value.

Besides, the alternative of hypothesis $H_{\text {alt }}$ can define as Equn.17 to counter the null hypothesis $H_{\text {null }}$.

$$
\begin{aligned}
& H_{\text {null }}: \mu_{\text {Proposed }}=\mu_{\text {MSPIN }}=\mu_{\text {SPIN-RL }}=\mu_{\text {SPIN-BC }} \\
& H_{\text {alt }}: \mu_{\text {Proposed }} \neq \mu_{\text {MSPIN }} \neq \mu_{\text {SPIN-RL }} \neq \mu_{\text {SPIN-BC }}
\end{aligned}
$$

To proceed with the ANOVA test, the number of a sample taken for validation is 5 for all schemes and the simulation scenario mentioned in section.6. Moreover, other critical metrics such as significance level value $\alpha=0.05$ and confidence interval $(\mathrm{CI})=95 \%$. The Table 2.(a) and 2.(b) have shown the input given to perform the ANOVA test for throughput and PDR and the output value of the test. Moreover, with confidence interval $=95 \%$, we have shown the output of the ANOVA test in Table 2.(a) and 2.(b). After the analysis of the test result and deception shown in Table 2, we can conclude the significance. It can be observed that 
the difference in the mean value of throughput has accepted are statistically significant, therefore we can reject the null hypothesis $H_{\text {null }}$ and accept the alternative hypothesis $H_{\text {alt }}$. Moreover, in our test for PDR, The f-ratio value is 0.83583 . The p-value is 0.493734 . Therefore, the ANOVA test result is not significant at $\mathrm{p}<0.05$ and $\mathrm{p}<0.10$. It also gives us the future roadmap to make the protocol efficient in term of PDR also. The detail description of ANOVA test, p-value, F-statistic and F-critical value are provided in [29], and we encourage reader interested in these statistical validation techniques.

Table 2(a). Summary of input

\begin{tabular}{lccccc}
\hline \multicolumn{7}{c}{ ANOVA test on throughput } \\
\hline Algorithms & Proposed & M-SPIN & SPIN-RL & SPIN-BC & Total \\
Count & 5 & 5 & 5 & 5 & 25 \\
$\sum \mathrm{X}$ & 3.99 & 3.12 & 2.885 & $\mathrm{n} / \mathrm{a}$ & 15.425 \\
Mean & 0.798 & 0.624 & 0.577 & $\mathrm{n} / \mathrm{a}$ & 0.617 \\
$\sum \mathrm{X}^{2}$ & 3.1881 & 2.02 & 1.717 & $\mathrm{n} / \mathrm{a}$ & 9.9861 \\
Std.Dev. & 0.0317 & 0.1352 & 0.1144 & $\mathrm{n} / \mathrm{a}$ & 0.1398 \\
\hline \multicolumn{7}{c}{ Output of ANOVA-Test For throughput } \\
\hline Source & $\mathrm{SS}$ & $\mathrm{df}$ & $\mathrm{MS}$ & \\
Between-treatments & 0.2268 & 4 & 0.0567 & $\mathrm{~F}=4.68442$ \\
Within-treatments & 0.2421 & 20 & 0.0121 & & \\
Total & 0.4689 & 24 & & & \\
\hline
\end{tabular}

The $\mathrm{f}$-ratio value is 4.68442 . The $\mathrm{p}$-value is .007855 . The result is significant at $\mathrm{p}<.05$

Table 2(b). Summary of input

\begin{tabular}{|c|c|c|c|c|c|}
\hline \multicolumn{6}{|c|}{ ANOVA test on PDR } \\
\hline Algorithms & Proposed & M-SPIN & SPIN-RL & SPIN-BC & Total \\
\hline Count & 5 & 5 & 5 & 5 & 20 \\
\hline$\sum \mathrm{X}$ & 3.97 & 3.76 & 3.44 & 3.39 & 14.56 \\
\hline Mean & 0.794 & 0.752 & 0.688 & 0.678 & 0.728 \\
\hline$\sum \mathrm{X}^{2}$ & 3.1827 & 2.8888 & 2.463 & 2.3985 & 10.933 \\
\hline Std.Dev. & 0.0873 & 0.1238 & 0.1551 & 0.1582 & 0.1325 \\
\hline \multicolumn{6}{|c|}{ Output of ANOVA-Test For PDR } \\
\hline Source & SS & df & MS & \multirow{4}{*}{\multicolumn{2}{|c|}{$F=0.83583$}} \\
\hline Between-treatments & 0.0452 & 3 & 0.0151 & & \\
\hline Within-treatments & 0.2882 & 16 & 0.018 & & \\
\hline Total & 0.3333 & 19 & & & \\
\hline
\end{tabular}

The $\mathrm{f}$-ratio value is 0.83583 . The $\mathrm{p}$-value is .493734 . The result is not significant at $\mathrm{p}<.05$ and $\mathrm{p}<.10$ 


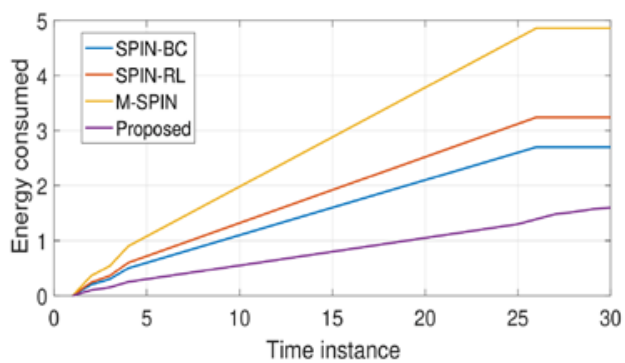

Fig. 6. Area of dimension $150 * 150 \mathrm{~m}^{2}$.

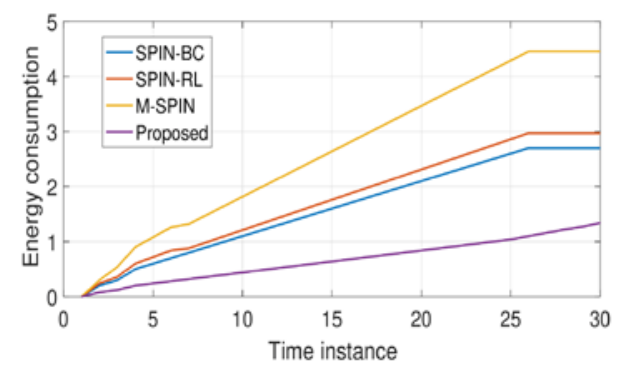

Fig. 7. Area of dimension $300 * 300 \mathrm{~m}^{2}$.

Total energy consumption by 150 sensor node

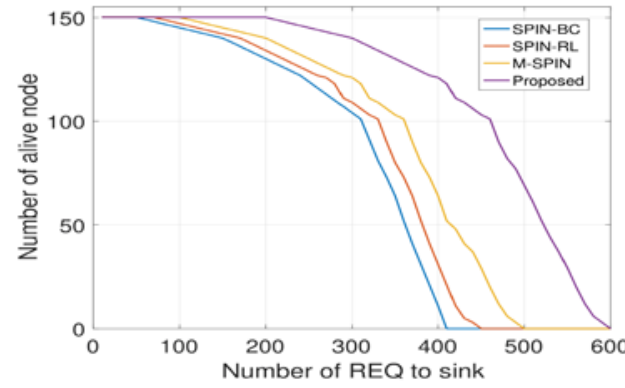

Fig. 8. Target area of dimension $150 * 150 \mathrm{~m}^{2}$.

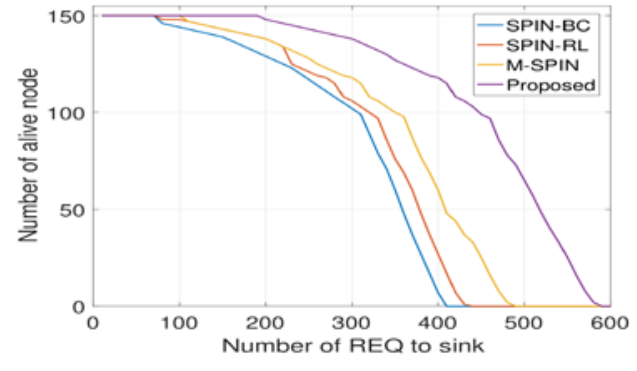

Fig. 9. Target area of dimension $300 * 300 \mathrm{~m}^{2}$ Number of alive node out of 150 sensors

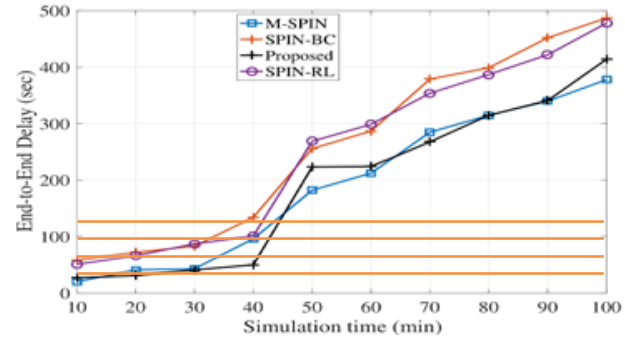

Fig. 10. Taget area of dimension $150 * 150 \mathrm{~m}^{2}$.

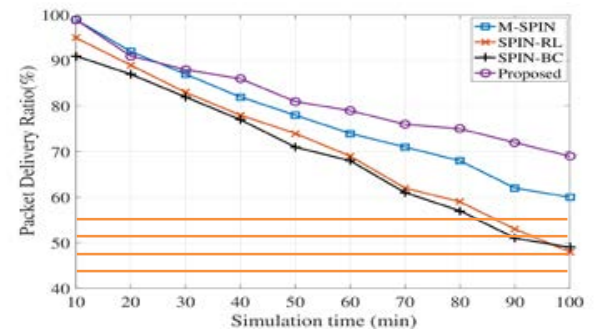

Fig. 11. Target area of dimension $300 * 300 \mathrm{~m}^{2}$. 
Average End-to-End packet delay

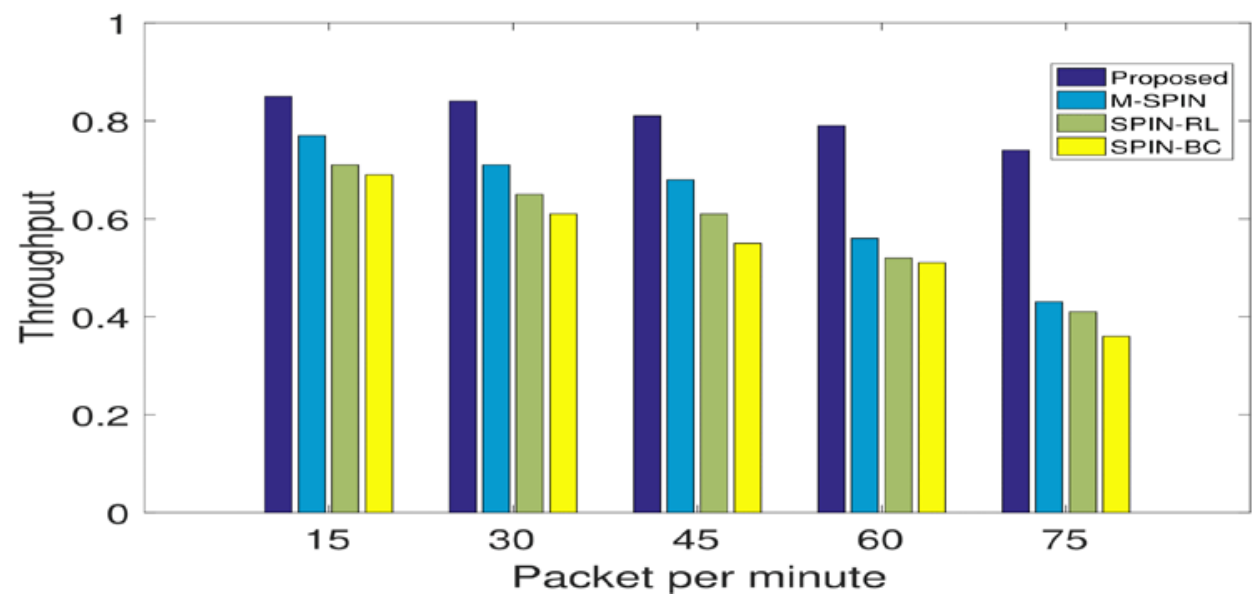

Fig. 12. Average throughput respect to packet generation rate for 150 sensor in target area of dimension $300 * 300 \mathrm{~m}^{2}$.

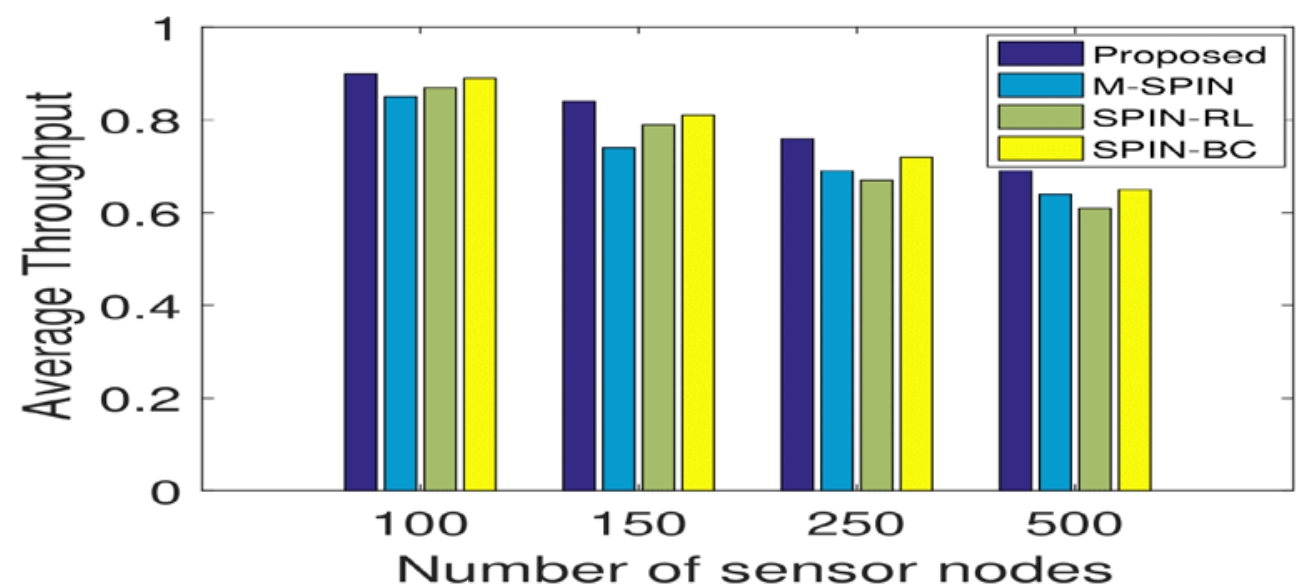

Fig. 13. Average throughput in low traffic scenario.

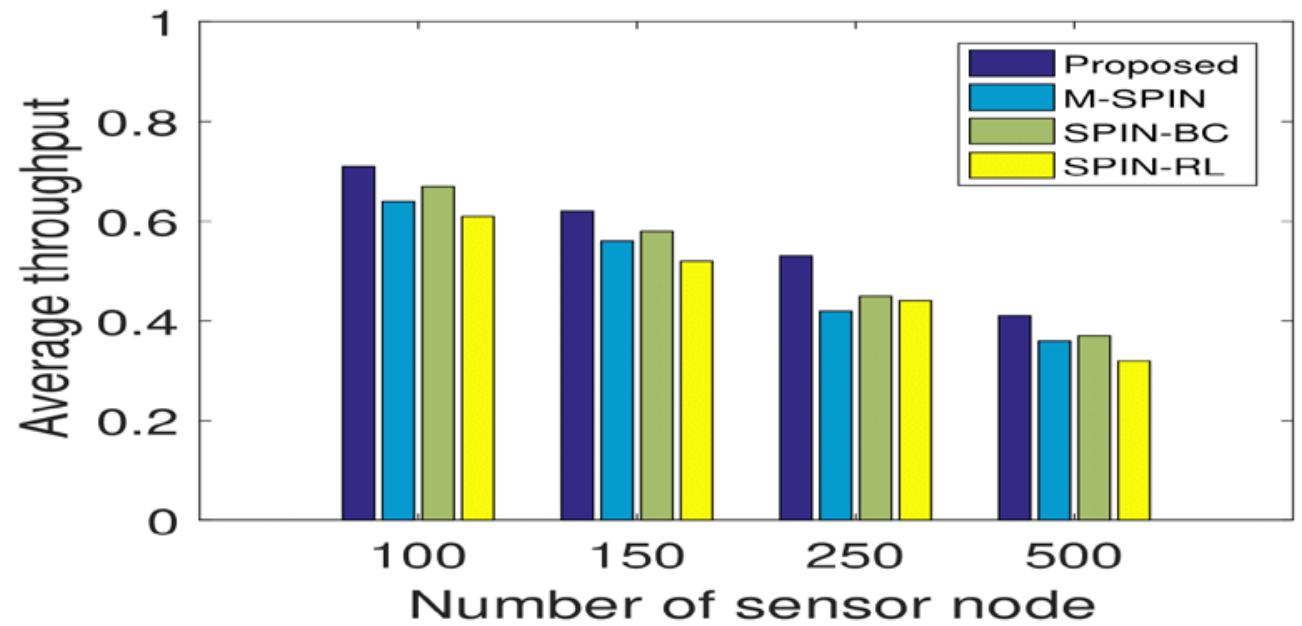

Fig. 14. Average throughput in medium traffic scenario. 


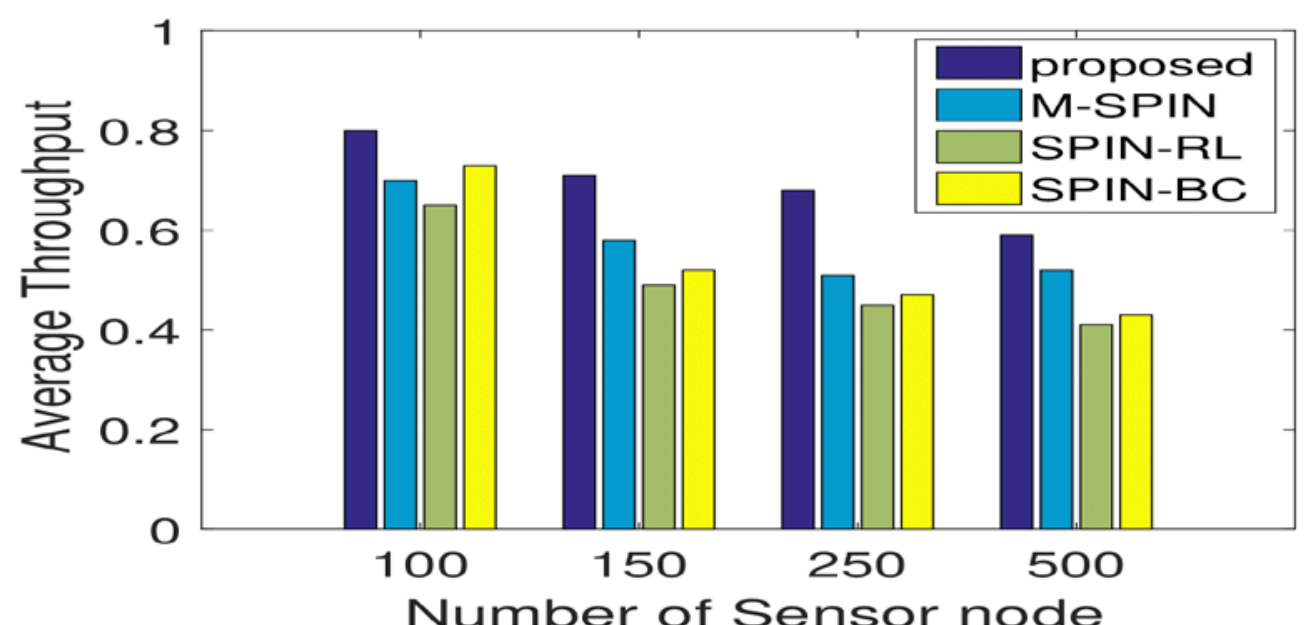

Fig. 15. Average throughput in high traffic scenario.

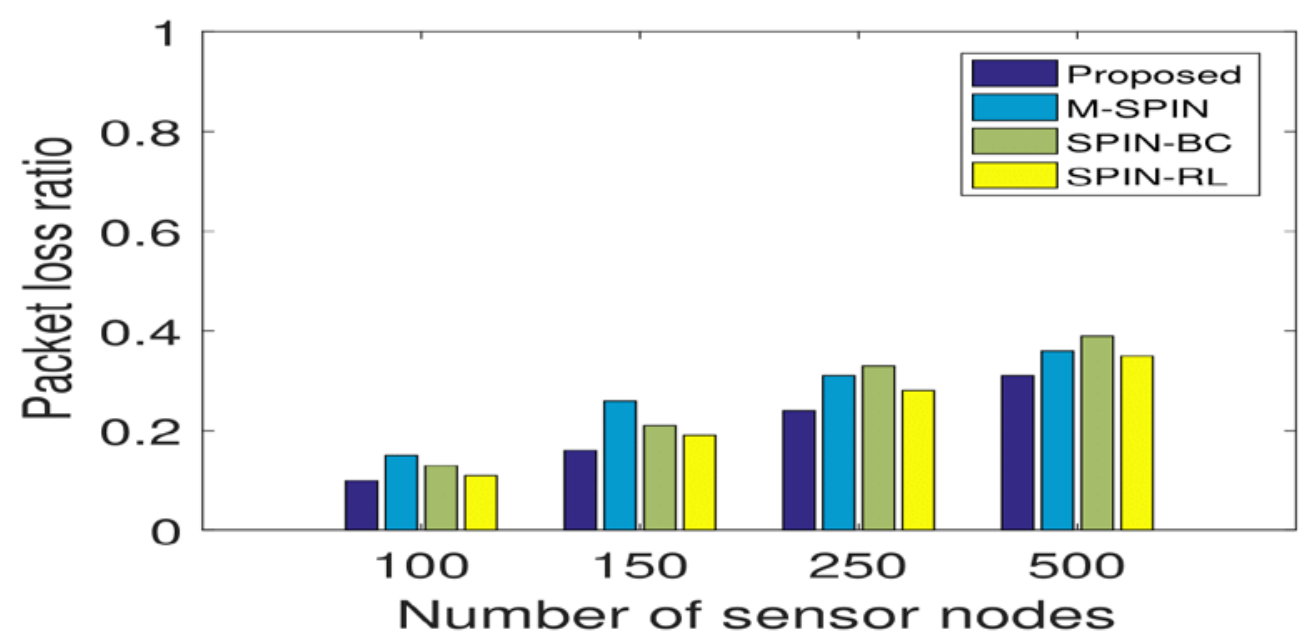

Fig. 16. Average End-to-end delay in low traffic scenario.

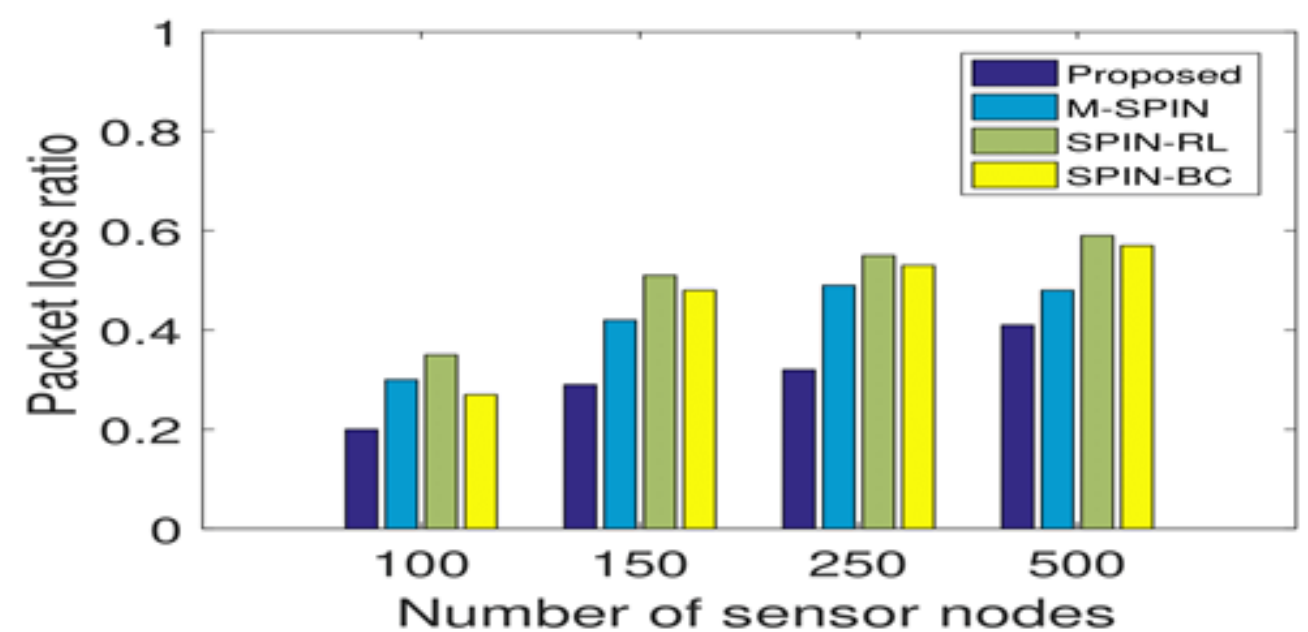

Fig. 17. Average End-to-end delay in medium traffic scenario. 


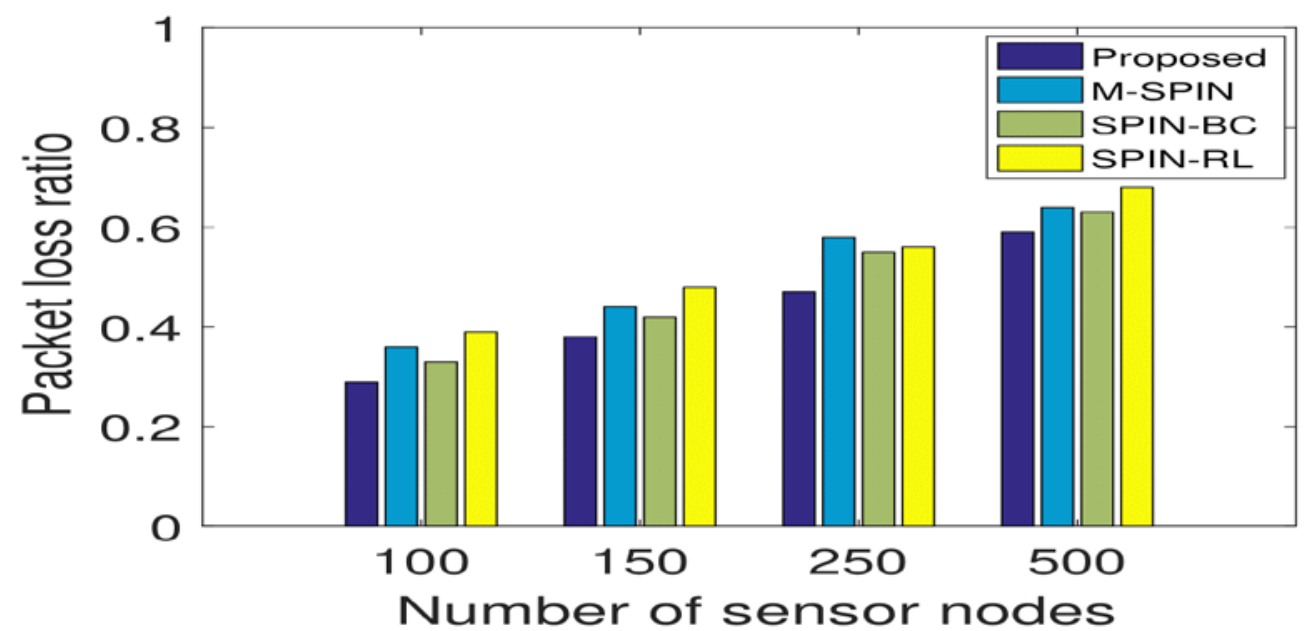

Fig. 18. Average End-to-end delay in high traffic scenario.

\section{Conclusion}

The proposed routing protocol is suitable for event-based application environments where quick response is of importance. In the initial stage of the proposal distance of each deployed node from the sink is calculated and is stored by a node for further references. The hop distance calculation process is a decentralized method that drops the duplicate packed if the hop distance of the transmitting node is greater than the receiving node. In the data transmission phase, a neighbor node decides to forward the data from the source node based on its available energy. If the energy is above the predefined threshold REQ message is sent along with current energy level and hop distance information. Source node after receiving the REQ packet from neighbor sorts the node based on energy level and hop distance and then the node with maximum energy level and min hop count is selected for data forwarding. Comparisons with other states of the art technique like SPIN-BC, SPIN-RL, and M-SPIN reveals the effectiveness of the proposal in terms of network lifetime and data throughput. Moreover, the given proposal has analysed the parametres such as network lifetime, packet delay, throughput etc. for the application scenario where the topology is uniform in nature. There was a study on the random topology of wireless sensor nodes and its effect on accuracy as well as energy consumption [26]. The application where nodes are mobile in nature also crete the random topologu over time. Moreover, for random topology clustered point processes based on a different mathematical model such as Gauss-Poisson process (GPP) [27], Neyman-Scott process [28] also available which need a more extensive study for our future work.

Future work will be focused toward the enhancing the PDR of the proposal and optimum threshold identification using evolutionary algorithm. Another future direction would be the path identification in the quadrant where the source node is located, helping in proper load distribution of incoming traffic toward the sink node. The effecctivness of the proposal in a realistic secnario like vehicular ad-hoc or mobile ad-hoc network will also be a challenge and can be attemted in futture studies. 


\section{References}

[1] Yue, Y.-G., He, P., “A comprehensive survey on the reliability of mobile wireless sensor networks: Taxonomy, challenges, and future directions,” Information Fusion, 44, 188-204, 2018. Article (CrossRef Link)

[2] Rashid, B., Rehmani, M.H., “Applications of wireless sensor networks for urban areas: A survey,” Journal of network and computer applications, 60, 192-219, 2016. Article (CrossRef Link)

[3] Yetgin, H., Cheung, K.T.K., El-Hajjar, M., Hanzo, L.H., "A survey of network lifetime maximization techniques in wireless sensor networks," IEEE Communications Surveys \& Tutorials, 19(2), 828-854, 2017. Article (CrossRef Link)

[4] Yang, X., Deng, D., Liu, M., “An overview of routing protocols on wireless sensor network,” in Proc. of 2015 4th International Conference on Computer Science and Network Technology (ICCSNT), vol. 1, pp. 1000-1003, 2015. Article (CrossRef Link)

[5] Echoukairi, H., Bourgba, K., Ouzzif, M., “A survey on at routing protocols in wireless sensor networks,” in Proc. of International Symposium on Ubiquitous Networking, pp. 311-324, 2015. Article (CrossRef Link)

[6] Mansouri, D., Mokdad, L., Ben-othman, J., Ioualalen, M., "Dynamic and adaptive detection method for ooding in wireless sensor networks," International Journal of Communication Systems, 30(12), 3265-3276, 2017. Article (CrossRef Link)

[7] Sharma, G., Bala, S., Verma, A.K., "Comparison of flooding and directed diffusion for wireless sensor network,” in Proc. of 2009 Annual IEEE India Conference, pp. 1-4, 2009. Article (CrossRef Link)

[8] Pantazis, Nikolaos A., Stefanos A. Nikolidakis, and Dimitrios D. Vergados. "Energy-efficient routing protocols in wireless sensor networks: A survey," IEEE Communications surveys \& tutorials, 15(2), 551-591, 2013. Article (CrossRef Link)

[9] Liu, T., Li, Q., Liang, P., “An energy-balancing clustering approach for gradient-based routing in wireless sensor networks,” Computer Communications, 35(17), 2150-2161, 2012. Article (CrossRef Link)

[10] Liu, X., “A survey on clustering routing protocols in wireless sensor networks,” sensors, 12(8), 11113 - 11153, 2012. Article (CrossRef Link)

[11] Singh, K., "Wsn leach based protocols: A structural analysis," in Proc. of 2015 International Conference and Workshopon Computing and Communication (IEMCON), pp. 1-7, 2015. Article (CrossRef Link)

[12] Ferng, H.-W., Tendean, R., Kurniawan, A., "Energy-efficient routing protocol for wireless sensor networks with static clustering and dynamic structure," Wireless Personal Communications, 65(2), 347-367, 2012. Article (CrossRef Link)

[13] Mahakud, R., Rath, S., Samantaray, M., Sinha, B., Priya, P., Nayak, A., Kumari, A., “Energy management in wireless sensor network using pegasis,” Procedia Computer Science, 92, 207212, 2016. Article (CrossRef Link)

[14] Nighot, M., Ghatol, A., "Gps based distributed communication protocol for static sensor network (gdcp),” Procedia Computer Science, 78, 530-536, 2016. Article (CrossRef Link)

[15] Oller, J., Demirkol, I., Casademont, J., Paradells, J., Gamm, G.U., Reindl, L., "Has time come to switch from duty-cycled mac protocols to wake-up radio for wireless sensor networks?," IEEE/ACM Transactions on Networking, 24(2), 674-687, 2015. Article (CrossRef Link)

[16] Sohrabi, K., Gao, J., Ailawadhi, V., Pottie, G.J., "Protocols for self-organization of a wireless sensor network,” IEEE personal communications, 7(5), 16-27, 2000. Article (CrossRef Link)

[17] Arora, N., "Performance analysis of aodv, dsr and zrp in manets using qualnet simulator," Journal of Engineering Science and Technology Review, 6(1), 21-24, 2013. Article (CrossRef Link)

[18] Dunkels, A., Osterlind, F., He, Z., “An adaptive communication architecture for wireless sensor networks,” in Proc. of the 5th International Conference on Embedded Networked Sensor Systems, pp. 335-349, 2007. Article (CrossRef Link) 
[19] Arya, R., Sharma, S., “Optimization approach for energy minimization and bandwidth estimation of wsn for data centric protocols,” International Journal of System Assurance Engineering and Management, 9(1), 2-11, 2018. Article (CrossRef Link)

[20] Perrig, A., Szewczyk, R., Tygar, J.D., Wen, V., Culler, D.E., "Spins: Security protocols for sensor networks," Wireless networks, 8(5), 521-534, 2002. Article (CrossRef Link)

[21] Maroti, M., Kusy, B., Simon, G., Ledeczi, A., “The flooding time synchronization protocol," in Proc. of the 2nd International Conference on Embedded Networked Sensor Systems, pp. 39-49, 2004. Article (CrossRef Link)

[22] Peng, S., Low, C., "Energy neutral directed diffusion for energy harvesting wireless sensor networks,” Computer Communications, 63, 40-52, 2015. Article (CrossRef Link)

[23] Liu, H.-H., Su, J.-J., Chou, C.-F., "On energy-efficient straight-line routing protocol for wireless sensor networks,” IEEE systems journal, 11(4), 2374-2382, 2015. Article (CrossRef Link)

[24] Hayes, T., Ali, F.H., "Proactive highly ambulatory sensor routing (phaser) protocol for mobile wireless sensor networks,” Pervasive and Mobile Computing, 21, 47-61, 2015. Article (CrossRef Link)

[25] Yadav, S.S., Chitra, A., Deepika, C.L., "Reviewing the process of data fusion in wireless sensor network: a brief survey," International Journal of Wireless and Mobile Computing, 8(2), 130140, 2015. Article (CrossRef Link)

[26] Zabini, F., Calisti, A., Dardari, D., \& Conti, A., "Random sampling via sensor networks: Estimation accuracy vs. energy consumption," in Proc. of 2016 24th European Signal Processing Conference (EUSIPCO). Presented at the 2016 24th European Signal Processing Conference (EUSIPCO), 2016. Article (CrossRef Link)

[27] Guo, A., Zhong, Y., Zhang, W., \& Haenggi, M., "The Gauss-Poisson Process for Wireless Networks and the Benefits of Cooperation,” IEEE Transactions on Communications, 64(5), 1916-1929, 2016. Article (CrossRef Link)

[28] R. K. Ganti and M. Haenggi, "Interference and outage in clustered wireless ad hoc networks," IEEE Trans. Inf. Theory, vol. 55, no. 9, pp. 4067-4086, Sep. 2009. Article (CrossRef Link)

[29] Shapiro, Samuel Sanford, and Martin B. Wilk, "An analysis of variance test for normality (complete samples),” Biometrika, 52(3/4), 591-611, 1965. Article (CrossRef Link) 


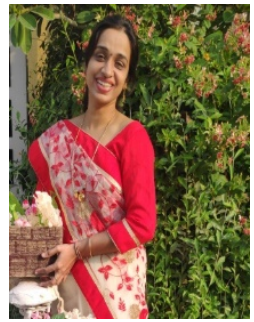

Chaya.Shivalingagowda, Currently Ph. D Research scholar in GITAM Vizag from the Department of Electronics \& Communication. She is an Assistant professor at Kalsekar Engineering College, New Panvel, Mumbai. She completed a Master in Engineering at Mumbai University, Mumbai, with Distinction. She has published multiple international journal and conference proceedings in IEEE and Springer. She also served as Spoken Tutorial Coordinator for College Conducting various FOSSEE Courses. Given FDP and seminars on Open Source like Scilab, Ns2 Technical Training for Placements in various engineering colleges in Mumbai. She is a member of UACEE, IETE, IWSA. Research areas are Optical communication and Wireless Sensor Network.

E-Mail information: chaya.ece@gmail.com and chaya.ravindra@gmail.com

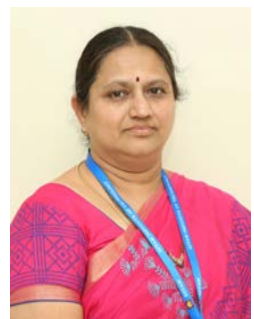

Dr.P.V.Y.Jayasree received her M.E. and Ph.D. degrees in Electronics and Communications Engineering from Andhra University, Visakhapatnam and JNT University, Kakinada in 1999 and 2010 respectively. She is currently a Professor and Head of the Department of Electronics and Communications Engineering with GITAM University, Visakhapatnam, India. Her research interest lies in the areas of EMI/EMC, Antennas, Microwaves, etc.

E-Mail information:

jayasree.pappu@gitam.edu and ece.pjayasree@gmail.com

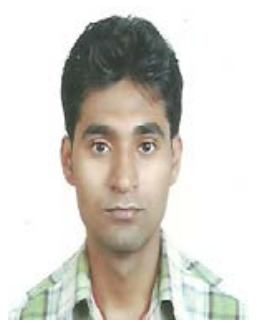

Dinesh K Sah, currently Ph. D research scholar (Full-Time) in the Indian Institute of Technology(ISM), Dhanbad from the Department of Computer Science \& Engineering. His research areas are Wireless Sensor Networks, optical network, 5G technologies, and network security.

E-Mail Information: dksah.iitd@gmail.com 